\title{
Patient Selection Factors for Stereotactic Body Radiotherapy and Radioembolization for Hepatic Metastases
}

\section{TE Schefter}

Department of Radiation Oncology, University of Colorado School of Medicine,1665 Aurora Ct, Suite 1032 MS F-706, Aurora, CO 80045, USA

\begin{abstract}
Liver metastases are a common source of morbidity and mortality. Despite significant improvements in outcome with systemic therapy in recent years, it is infrequent that liver metastases or sites of macrometastatic disease are controlled permanently. Furthermore, most liver metastases are not amenable to what is considered the gold standard locally ablative therapy, surgical resection and consequently there has been a surge in interest in non surgical ablative therapies. Review of patient selection factors for two such liver directed radiation therapies, radioembolization and stereotactic body radiation therapy (SBRT) will be addressed.
\end{abstract}

\section{Introduction}

\section{Making the Case for Liver Directed Therapy}

Colorectal cancer (CRC), the most common source of liver metastases, is also the most common gastrointestinal (GI) malignancy and represents the paradigm model for locally ablative therapies for metastatic disease. Like most GI cancers spread to the liver is via the portal vein. Liver metastases occur approximately 50 percent of the time in the natural history of colorectal cancer and the vast majority of these cases are not amenable to surgical resection [1] . Highly selected patients with limited CRC liver metastases and without extra-hepatic disease can expect 5 year overall survival rates as high as 40 to 60 percent with surgical resection [1-3].

Noncolorectal nonneuroendocrine (NCRNE) liver metastases generally portend a much poorer prognosis and therefore practices have been quite variable. Recently, Duke investigators published a retrospective report of a decade of experience with resection for solid primaries and showed that while liver resection for NCRNE liver metastases had slightly worse prognosis compared to CRC and neuroendocrine (NE) liver metastases, the actuarial 5 year overall survival rate was $37 \%$ [4]. While this was a retrospective study involving a highly selected and heterogeneous group, the favorable overall outcome provides support for liver directed therapy for NCRNE liver metastases as well.

Liver metastases from breast cancer are associated with an adverse prognosis as they generally don't respond as well to systemic therapy compared to bone and other soft tissue metastases. Liver directed locally ablative therapies for liver metastases in combination with systemic therapy are therefore attractive. A recent review of the literature identified a huge variation in median survival (27 to 63 months) following resection of breast cancer liver metastases [5] Further confounding the impact of surgical resection from retrospective studies of breast cancer liver metastases is the tremendous variability in systemic therapy. Controlling for this is impossible outside a formal prospective clinical trial.

Liver metastases are extremely common but most patients are not amenable to surgical resection, either for medical, disease extent or surgical reasons. Nonsurgical locally ablative therapies are therefore desirable. These therapies are either noninvasive or minimally invasive compared to surgery and if equally effective might even represent a desirable alternative to surgery for patients with resectable liver metastases.

\section{Oligometastases}

Hellman et al speculated that there was an intermediary "oligometastatic" state between localized and widely metastatic that was potentially curable [5]. Oligometastases are defined as minimal burden metastatic disease with limited organs of involvement and overall number of metastases. There is no absolute burden of disease that defines the oligometastatic state but 5 to 6 metastases are generally accepted. Increased awareness combined with advancements in imaging in the last decade has resulted in improved detection of oligometastases. Parenthetically, Hellman recognized the potential role of radiation therapy to cure metastatic disease more than 20 years ago. It is only in the last 5 years that radiation oncologists are fully appreciating this prediction. For example, in a 2011 United States SBRT use survey, $63.9 \%$ of radiation oncologists were using SBRT and $54.5 \%$ of the treatments were for liver tumors (mostly metastases) [6].

In terms of prognosis, it is important to distinguish de novo oligometastases from systemic therapy induced oligometastases, as the former have a much better outlook. This primarily relates to the risk of metastatic progression, with the latter category being at much higher risk $[7,8]$. Another factor potentially contributing to worse outcome is that response to radiation therapy is likely to be better for relatively chemotherapy/treatment naïve disease, as tumors are less likely to have acquired therapy resistant clones.

Stanford investigators have been exploring the use of positron emission tomography (PET) to quantify metastatic disease burden and they have consistently shown that metabolic tumor burden (MTB) as measured by PET is an independent predictor of overall outcome $[9,10]$. Whether interventions such as SBRT or selective internal radiation therapy (SIRT) to sites of gross metastatasis(es) in order

*Corresponding author: TE Schefter, Department of Radiation Oncology, University of Colorado School of Medicine, 1665 Aurora Ct, Suite 1032 MS F-706 Aurora, CO 80045, USA, Tel: 720-848-0116; Fax: 720-848-0239; E-mail: Tracey. schefter@ucdenver.edu

Received June 28, 2011; Accepted December 04, 2011; Published December 06, 2011

Citation: Schefter TE (2011) Patient Selection Factors for Stereotactic Body Radiotherapy and Radioembolization for Hepatic Metastases. J Nucl Med Radiat Ther 2:119. doi:10.4172/2155-9619.1000119

Copyright: (c) 2011 Schefter TE. This is an open-access article distributed unde the terms of the Creative Commons Attribution License, which permits unrestricted use, distribution, and reproduction in any medium, provided the original author and source are credited. 
to keep the metastatic burden below some threshold,[11] imparts a survival advantage is an area of ongoing research.

Currently, locally ablative radiation therapy for oligometastatic disease represents a very significant proportion of radiation oncology practice and therefore careful selection of patients most likely to benefit is crucial.

\section{Radiation Therapy}

In the past, radiation therapy for liver metastatases was considered solely for palliation, as radiation doses that could be safely delivered with crude techniques were far less than required for ablation. Advancements in radiation planning and delivery, allowing highly conformal radiation treatment, caused resurgence in its use as higher doses could be given safely. SBRT is a form of 3-dimensional conformal external beam radiotherapy (3-D CRT) which uses stereotactic localization, respiratory motion control and image-guided relocalization. A full account of the technical aspects of SBRT is beyond the scope this paper but interested readers can refer to summary reports. $[12,13]$

\section{SBRT}

The dose-limiting toxicity from whole liver external beam radiation treatment is radiation-induced liver disease (classic RILD), initially called radiation hepatitis [1] and characterized pathologically by central vein occlusion, as described by Reed and Cox.[1 ] With the advent of 3-D CRT planning and delivery technology that allowed for partial liver irradiation, it was recognized that higher tumor doses more likely to cause tumor ablation could be delivered safely, as long as the mean dose to the liver was kept to less than safely tolerated whole liver doses [16,17]. SBRT is a more specialized form of 3-D CRT, defined in the United States as 5 or fewer high dose per fractions (daily treatments) of radiation treatment, which are delivered in a total time of one to two weeks $[14,15]$. This is much shorter than the overall time for conventionally fractionated external beam radiotherapy. Unless internal fiducials (required only when using Cyberknife) are placed to aid with target delineation and daily treatment target re-localization, SBRT is completely non invasive. Typical total doses for SBRT are in the range of 40 to 60 Gy delivered in 1 to 5 fractions. This represents doses per fraction of 8 to 20 Gy versus 1.8 to 2 Gy per fraction for conventionally fractionated treatment.

Published reports have demonstrated 2 year actuarial local control rates ranging from 50 to $100 \%$, [18-21] with the best control published thus far coming from Rule et al using $60 \mathrm{~Gy}$ in 5 fractions. Toxicity from SBRT has been minimal for patients with no underlying cirrhotic liver disease. The most common morbidity observed is mild fatigue, chest wall pain and gastrointestinal morbidity.

Studies to date have been small and confounded by broad eligibility criteria including varying primary sites, variable size and number of metastases and degree of prior systemic therapy. Other than radiation dose, which has clearly been shown to correlate with local control, it is difficult to draw conclusions about other prognostic factors based on the studies reported to date. Future trials of RT for liver metastases will require narrower eligibility criteria while controlling for the intensity of pre RT systemic therapy to further clarify prognostic factors that are correlated with local control and overall outcome.

\section{Radioembolization}

Radioembolization (RE), also called selective internal radiation therapy (SIRT), is a form of brachytherapy (internal radiation therapy) and is to be distinguished from SBRT which is a form of teletherapy or external beam radiation therapy. Radioembolization is the focus of all reports in these 2 special issues and therefore an exhaustive review other than as it relates to patient selection will not be addressed. The focus here will be on patient selection factors for SBRT versus or in combination with radioembolization.

Briefly, RE has been shown to be safe and effective for liver metastases from CRC and Non CRC solid primaries [22-28]. Based on a compilation of studies to date, Riad and colleagues summarized the first reporting guideline document for studies of RE [29]. As studies ensue, investigators will have standardized reporting guidelines for patient selection, treatment methodology and imaging follow-up and this will facilitate clearer communication between investigators and comparison between studies.

\section{Sbrt Versus Radioembolization}

SBRT is a focal liver treatment, much like radiofrequency ablation (RFA) and surgical resection and therefore more limited burden disease is best suited for SBRT. SBRT has the advantage over other partial liver treatments like RFA, selective RE and surgery because it is completely non invasive. What defines "limited burden" disease is somewhat controversial, however 3 or fewer metastases is a reasonable guide. Beyond 3 metastases, even if one could spare enough normal liver to minimize the risk of radiation induced liver dysfunction as a result of SBRT, the risk of intrahepatic progression is significant. Consequently, more comprehensive treatment coverage of the liver using RE is attractive.

There are advocates of RE for all cases of liver metastases, regardless of extent of involvement, as long as there are no contraindications such as total bililrubin $>2 \mathrm{mg} / \mathrm{dL}$ or portal vein thrombosis. For limited disease that has traditionally been considered more amenable to SBRT, RFA or surgery, proponents of RE are exploring selective and super selective RE. Selective techniques are much more operator dependant and generally involve treating less than a liver lobe, sometimes even just one or two segments. One can contend however that SBRT is more advantageous for confined or limited disease as generally more liver can be spared because it is not necessary to treat all vascular segments spanned by the tumor(s). The other advantage of SBRT over RE is in being able to deliver much more accurate and precise radiation dose. While there have been efforts to characterize dose distributions obtained or delivered with RE, it is not nearly as well defined and precise as dosimetry with external beam radiation therapy. Furthermore, RE relies on tumor vascular supply for delivery of both radiation and embolic aspects of RE therapy. Large lesions with poor blood flow to all or parts of the tumor, especially centrally, are unlikely to be targeted appropriately with RE, thus another scenario where SBRT might be preferred.

Bevacizumab and other vascular endothelial growth factor (VEGF) inhibitors cause reduced blood flow to hepatic metastases, a result of vascular pruning and while it can be reversible after a minimum of 6 weeks off therapy, limited disease that is conducive to SBRT might be more appropriately treated that way rather than waiting for the vascular effects to normalize. A break in therapy of 2 months might result in significant tumor progression, rendering less favorable outcome.

\section{Sbrt Plus Radioembolization}

Occasionally it might be desirable to combine SBRT and RE, either as planned combined sequential treatment or offering one therapy following intrahepatic failure after the other. While previous 
hepatic radiation is considered a relative contraindication to $\mathrm{RE}$, this is generally limited to whole liver or significant volume prior liver irradiation [30]. Often, there are significant delays to administering $\mathrm{RE}$, either a result of health insurance issues or practical limitations of coordinating a multi-disciplinary treatment. Whatever the reason it may be reasonable to treat up to 4 or 5 metastases with SBRT if it can be done while respecting conservative dose volume histogram constraints, as a temporizing measure or bridge to RE.

The most common indication for the unplanned combination radiation therapies occurs following SBRT as the most common site of progression following liver SBRT for oligometastatic liver metastases is the liver. As long as liver function remains near normal, these patients can be safely treated with salvage RE (Schefter T, unpublished data). It is also reasonable to consider salvage liver SBRT for limited burden recurrence following whole liver RE. These patients typically have limited therapeutic options.

\section{Conculsions}

In the past metastatic disease, especially anything more than solitary metastases were considered for palliative radiation only. We now know that oligometastatic liver disease represents a state where locally ablative therapies can potentially confer improvements in overall outcome. RE and SBRT are two forms of radiation therapy, the former is brachytherapy and is best reserved for patients with vascular hepatic metastases more extensive than is appropriate for SBRT and the latter is a form of external beam radiation therapy and is preferred for patients with more limited oligometastatic disease. Future trials will further characterize patient and tumor selection factors favoring RE or SBRT or both.

\section{References}

1. Simmonds PC, Primrose JN, Colquitt JL, Garden OJ, Poston GJ, et al. (2006) Surgical resection of hepatic metastases from colorectal cancer: a systematic review of published studies. Br J Cancer 94: 982-999.

2. Fong Y, Fortner J, Sun RL, Brennan MF, Blumgart LH. (1999) Clinical score for predicting recurrence after hepatic resection for metastatic colorectal cancer: analysis of 1001 consecutive cases. Ann Surg 230: 309-318.

3. House MG, Ito H, Gönen M, Fong Y, Allen PJ, et al. (2010) Survival after hepatic resection for metastatic colorectal cancer: trends in outcomes for 1,600 patients during two decades at a single institution. J Am Coll Surg 210: 744 745

4. Reddy SK, Barbas AS, Marroquin CE, Morse MA, Kuo PC, et al. (2007) Resection of noncolorectal nonneuroendocrine liver metastases: a comparative analysis. J Am Coll Surg 204: 372-382.

5. Hellman S, Weichselbaum RR. (2005) Importance of local control in an era of systemic therapy. Nature Clinical Practice Oncology 2: 60-61.

6. Pan H, Simpson DR, Mell LK, Mundt AJ, Lawson JD (2011) A survey of stereotactic body radiotherapy use in the United States. Cancer 117: 45664572

7. Milano MT, Katz AW, Okunieff P (2010) Patterns of Recurrence After CurativeIntent Radiation for Oligometastases Confined to One Organ. Am J Clin Onc 33: $157-163$

8. Milano MT, Philip A, Okunieff P. (2009)Analysis of patients with oligometastase undergoing two or more curative-intent stereotactic radiotherapy courses. Int $J$ Radiat Oncol Biol Phys 73: 832-837.

9. Lee P, Weerasuriya DK, Lavori PW, Quon A, Hara W, et al. ( 2007) Metabolic Tumor burden predicts for disease progression and death in lung cancer. Int $J$ Radiat Oncol Biol Phys 69: 328-333

10. La TH, Filion EJ, Turnbull BB, Chu JN, Lee P, et al. (2009) Metabolic Tumor burden predicts for recurrence and death in head and neck cancer. Int J Radiat Oncol Biol Phys 74: 1335-1341.

11. Norton L,Simon R (1986) The Norton-Simon Hypothesis revisited. Cance Treat Rep 70: 163-169

12. Potters L, Kavanagh B, Galvin JM, Hevezi JM, Janjan NA, et al. (2010) American Society for Therapeutic Radiology and Oncology (ASTRO) and American College of Radiology (ACR) practice guideline for the performance of stereotactic body radiation therapy. Int J Radiat Oncol Biol Phys 76: 326-332.

13. Dawson LA, Jaffray DA (2007) Advances in image-guided radiation therapy. J Clin Oncol 25: 938-46.

14. Ingold JA, Reed GB, Kaplan HS (1965) Radiation hepatitis. Am J Roentgenol 93: $200-208$

15. Reed GB, Cox AJ, J. Cox Jr (1966) The Human Liver after Radiation Injury. Am J Pathol 48: 597-611.

16. Dawson LA, Normolle D, Balter JM, Mc Ginn CJ, Lawrence TS, et al. (2002) Analysis of radiation-induced liver disease using the Lyman NTCP model. Int $J$ Radiat Oncol Biol Phys 53: 810-821.

17. Schefter TE, Kavanagh BD, Timmerman RD, Cardenes HR, Baron A et al. 2005) A Phase I trial of stereotactic body radiation therapy for liver metastases. Int J Radiat Oncol Biol Phys 62: 1371-1378.

18. Rusthoven KE, Kavanagh BD, Cardenes H, Stieber VW, Burri SH, et al. (2009) Mature Results of a Multi-Institutional Phase I/II Trial of Stereotactic Body Radiation Therapy for Liver Metastases. J Clin Oncol 27: 1572-1578.

19. Lee MT, Kim JJ, Dinniwell R, Brierley J, Lockwood G, et al.(2009)Phase I Study of Individualized Stereotactic Body Radiotherapy of Liver Metastases. J Clin Oncol 27: 1585-1591.

20. Rule W, Timmerman R, Tong L, Abdulrahman R, Meyer J, et al. ( 2010) Phase I Dose-Escalation Study of Stereotactic Body Radiotherapy in Patients With Hepatic Metastases. Ann Surg Oncol 18: 1081-1087.

21. Goodman KA, Wiegner EA, Maturen KE, Zhang Z, Mo Q, et al.( 2010) Doseescalation study of single-fraction stereotactic body radiotherapy for liver malignancies. Int J Radiat Oncol Biol Phys 78: 486-493.

22. Rhee TK, Lewandowski RJ, Liu DM, Mulcahy MF, Takahashi G, et al. (2008)90Y radioembolization for metastatic neuroendocrine liver tumors: preliminary results from a multiinstitutional experience. Ann Surg 247: 1029-1035.

23. Kennedy AS, Dezarn WA, McNeillie P, Coldwell D, Nutting C, et al.( 2008) Radioembolization for unresectable neuroendocrine hepatic metastases using resin 90Y-microspheres: early results in 148 patients. Am J Clin Oncol 31 271-279.

24. Sharma RA, Van Hazel GA, Morgan B, Berry DP, Blanshard K, et al. (2007) Radioembolization of liver metastases from colorectal cancer using yttrium-90 microspheres with concomitant systemic oxaliplatin, fluorouracil, and leucovorin chemotherapy. J Clin Oncol 25: 1099-1106.

25. Kennedy AS, McNeillie P, Dezarn WA, Nutting C, Sangro B, et al. (2009) Treatment parameters and outcome in 680 treatments of internal radiation with resin 90Y-microspheres for unresectable hepatic tumors. Int J Radiat Oncol Biol Phys 74: 1494-1500.

26. Mulcahy MF, Lewandowski RJ, Ibrahim SM, Sato KT, Ryu RK, et al. 2009) Radioembolization of colorectal hepatic metastases using yttrium-90 microspheres. Cancer 115: 1849-1858.

27. Van Hazel G, Blackwell A, Anderson J, Price D, Moroz P, et al. (2004) Randomised phase 2 trial of SIR-Spheres plus fluorouracil/leucovorin chemotherapy versus fluorouracil/leucovorin chemotherapy alone in advanced colorectal cancer. J Surg Oncol 88: 78-85.

28. Kennedy AS, Salem R (2010) Radioembolization (yttrium-90 microspheres) for primary and metastatic hepatic malignancies. Cancer J 16: 163-175.

29. Salem R, Lewandowski RJ, Gates VL, Nutting CW, Murthy R, et al. (2011) Research reporting standards for radioembolization of hepatic malignancies. $J$ Vasc Interv Radiol 22: 265-278.

30. Practice guideline for radioembolization with microsphere brachytherapy device (RMBD) for treatment of liver malignancies. 2008. www.acr.org 